\title{
The five-repetition sit-to-stand test as a functional outcome measure in COPD
}

\author{
Sarah E Jones, ${ }^{1}$ Samantha S C Kon, ${ }^{1}$ Jane L Canavan, ${ }^{1}$ Mehul S Patel, ${ }^{1}$ Amy L Clark, ${ }^{2}$ \\ Claire M Nolan, ${ }^{1}$ Michael I Polkey, ${ }^{1}$ William D-C Man ${ }^{1,2}$
}

${ }^{1} \mathrm{NIHR}$ Respiratory Biomedical Research Unit, Royal Brompton \& Harefield NHS Foundation Trust and Imperial College, Harefield, UK

${ }^{2}$ Harefield Pulmonary Rehabilitation Unit, Royal Brompton \& Harefield NHS Foundation Trust, Harefield, UK

\section{Correspondence to}

Sarah E Jones, Department of Respiratory Medicine and Pulmonary Rehabilitation, Harefield Hospital, Hill End Road, Harefield UB9 6JH, UK; s.jones5@rbht.nhs.uk

SEJ and SSCK contributed equally.

Received 15 March 2013 Revised 24 May 2013 Accepted 29 May 2013 Published Online First 19 June 2013

\begin{tabular}{l}
\hline To cite: Jones SE, Kon SSC, \\
Canavan JL, et al. Thorax \\
2013;68:1015-1020. \\
\hline
\end{tabular}

\section{ABSTRACT}

Background Moving from sitting to standing is a common activity of daily living. The five-repetition sit-tostand test (5STS) is a test of lower limb function that measures the fastest time taken to stand five times from a chair with arms folded. The 5STS has been validated in healthy community-dwelling adults, but data in chronic obstructive pulmonary disease (COPD) populations are lacking.

Aims To determine the reliability, validity and responsiveness of the 5STS in patients with COPD.

Methods Test-retest and interobserver reliability of the 5STS was measured in 50 patients with COPD. To address construct validity we collected data on the 5STS, exercise capacity (incremental shuttle walk (ISW)), lower limb strength (quadriceps maximum voluntary contraction (QMVC)), health status (St George's Respiratory Questionnaire (SGRQ)) and composite mortality indices (Age Dyspnoea Obstruction index (ADO), BODE index (BODE)). Responsiveness was determined by measuring 5STS before and after outpatient pulmonary rehabilitation (PR) in 239 patients. Minimum clinically important difference (MCID) was estimated using anchor-based methods.

Results Test-retest and interobserver intraclass correlation coefficients were 0.97 and 0.99 , respectively. 5STS time correlated significantly with ISW, QMVC, SGRQ, ADO and BBODE ( $r=-0.59,-0.38,0.35,0.42$ and 0.46 , respectively; all $p<0.001$ ). Median (25th, 75th centiles) 5STS time decreased with PR (Pre: 14.1 (11.5, 21.3) vs Post: $12.4(10.2,16.3) s ; p<0.001)$. Using different anchors, a conservative estimate for the MCID was $1.7 \mathrm{~s}$.

Conclusions The 5STS is reliable, valid and responsive in patients with COPD with an estimated MCID of $1.7 \mathrm{~s}$. It is a practical functional outcome measure suitable for use in most healthcare settings.

\section{INTRODUCTION}

Exercise performance captures the integrated and multisystemic effects of chronic obstructive pulmonary disease (COPD) and predicts adverse outcomes such as mortality. ${ }^{1}$ Although several laboratory-based and field tests have been validated in COPD, limitations exist which may prevent widespread use in some healthcare settings. Laboratory tests are expensive, labour intensive and require specialist equipment, personnel and space. Field walking tests are simpler and cheaper but are still not routinely used in primary, acute or home care settings as they are not practical in terms of space or time. For example, the 6 min walk test

\section{Key messages}

What is the key question?

- Is the five-repetition sit-to-stand test (5STS) a reliable and valid functional outcome measure in patients with chronic obstructive pulmonary disease (COPD)?

\section{What is the bottom line?}

- The 5STS shows excellent reliability, correlates with exercise capacity and lower limb strength and is responsive to pulmonary rehabilitation in COPD.

\section{Why read on?}

- The 5STS is a simple assessment tool that is feasible in all healthcare settings (including the home), and may be a rapid method of assessing changes in exercise capacity in COPD and screening for poor physical functioning individuals.

(6MWT) requires a $30 \mathrm{~m}$ flat course while the incremental shuttle walk (ISW) test requires a $10 \mathrm{~m}$ course; both require a repeat walk either on a different day or following adequate rest on the same day to account for learning effect. ${ }^{2} 3$ There is a need for reliable physical performance tests that are easy and quick to perform in most clinical settings, including the bedside.

The sit-to-stand (STS) manoeuvre is a common activity of daily living ${ }^{4}$ and is partly dependent on lower limb muscle function and balance. ${ }^{5} \quad 6$ Variations of the STS manoeuvre have been adapted as functional performance measures, including time taken to perform a given number of STS manoeuvres ${ }^{7}$ or the maximum number of STS manoeuvres in a given time period, usually 30 or 60 s. $^{8} 9$ These have been shown to correlate well with other objective physical performance measures such as Timed Up and Go, gait speed ${ }^{10}$ and the $6 \mathrm{MWT}^{9}$ in healthy older community-living populations as well as patients with stroke, Parkinson's disease and vestibular disorders.

The five-repetition STS test (5STS), which measures the time taken to stand five times from a sitting position as rapidly as possible, is the best described STS test in older adults. Normative values $^{7}$ and data on reliability ${ }^{11}$ and validity ${ }^{12}$ have been well described in healthy older 
community-dwelling individuals, but there is a paucity of data in COPD populations.

The aims of the study were therefore to establish (1) test-retest and interobserver reliability of the 5STS; (2) convergent validity, by demonstrating significant correlations with established measures of exercise capacity and lower limb muscle strength, health status and composite indices of mortality in patients with COPD; (3) discriminative validity, by demonstrating significantly reduced exercise capacity and lower limb muscle strength in patients unable to complete the 5STS; (4) responsiveness of the 5STS to intervention (ie, pulmonary rehabilitation (PR)); and (5) minimum clinically important difference (MCID) of the 5STS in patients with COPD. Our hypothesis was that 5STS time would correlate strongly with worsening exercise capacity and increasing quadriceps weakness and less strongly with health status and disease severity (as assessed by composite indices of mortality). We also hypothesised that the 5STS time would decrease significantly following PR and that this reduction in time would correlate significantly with improvement in an established field walking test.

\section{METHODS}

Subjects

Patients with stable COPD diagnosed according to the Global initiative for chronic Obstructive Lung Disease (GOLD) guidelines ${ }^{13}$ were recruited from outpatient respiratory and PR clinics at Harefield Hospital, UK. Exclusion criteria included exacerbation within preceding 4 weeks, lower limb joint surgery within preceding 3 months, unstable cardiac disease or predominant neurological limitation to walking (eg, significant hemiplegia). Some of the data have been previously presented in abstract form. ${ }^{14}$

\section{Measurement of the 5STS}

Measurements were made in an outpatient consultation room. A straight-backed armless chair with a hard seat was stabilised by placing it against a wall. Floor to seat height was $48 \mathrm{~cm}$. Seated participants were asked to come forward on the chair seat until the feet were flat on the floor and to fold their upper limbs across the chest. Participants were then instructed to stand up all the way and sit down once without using the upper limbs. For those unable to complete the initial manoeuvre or who required assistance, the test was terminated. If successful on the initial sit to stand, participants were then asked to stand up all the way and sit down landing firmly, as fast as possible, five times without using the arms. Timing with a stopwatch was started on the command 'go' and stopped at the end of the completed fifth stand; the time taken was recorded as the participant's score.

\section{Test-retest and interobserver reliability}

Fifty patients with COPD had a 5STS measurement on two occasions $24-48 \mathrm{~h}$ apart made by the same operator. The same 50 patients had 5STS measured simultaneously by two observers on the same occasion.

\section{Cross-sectional study}

5STS was measured in a convenience sample of 475 patients with COPD. Exercise capacity was measured using the ISW as previously described. ${ }^{3}$ The better of two walks after a minimum of $30 \mathrm{~min}$ rest was used for analysis. Lower limb strength was assessed by isometric maximum voluntary contraction of the quadriceps (QMVC) using a specially adapted chair fitted with a calibrated strain gauge ${ }^{15}$ and expressed as percentage predicted. ${ }^{16}$ Further measurements included the health-related quality of life St George's Respiratory Questionnaire (SGRQ), ${ }^{17}$ COPD Assessment Test (CAT), ${ }^{18}$ the Medical Research Council Dyspnoea scale (MRC) ${ }^{19}$ and spirometry. Composite indices of COPD mortality prediction, the BODE index $(\mathrm{iBODE})^{20}$ and Age Dyspnoea Obstruction index (ADO), ${ }^{21}$ were calculated. Comorbidities were recorded as the age-adjusted Charlson Index. ${ }^{22}$ Clinical characteristics of participants stratified according to the ability to complete the 5STS were described.

\section{Response to PR}

Three hundred and five patients in the cross-sectional convenience sample were subsequently referred for clinical reasons to an outpatient PR programme. The PR programme was an 8 -week multidisciplinary outpatient exercise and education programme comprising two supervised and at least one additional home session per week. The exercise training was individualised and included a mixture of aerobic and strength training. Initial walking exercise prescription was based on the outcome of the ISW and speed set at $80 \%$ of predicted peak oxygen consumption while the initial cycling prescription was based on symptom scores. Workloads and duration of exercise were increased through the programme as tolerated. 5STS, ISW and SGRQ were prospectively measured and data before and after PR compared. Participants unable to attempt or complete five stands or unable to complete the test within $1 \mathrm{~min}$ were automatically assigned a score of $60 \mathrm{~s}$.

\section{Minimum clinically important difference}

To determine the MCID of 5STS, anchor-based approaches were used. These compare the change in the outcome of interest against the change in another outcome measure, considered an anchor or external criterion. ${ }^{23}$ This is typically the response to a global assessment rating or a validated outcome measure. In this study we examined the median 'within patient' change in the 5STS against the following anchors: 'feeling much better' or 'better' on a 5-point Likert scale (ranging from 1 (feeling much better) to 5 (feeling much worse), as previously described $)^{18}$; a 'within patient' achievement of the MCID for the ISW (>47.5 $\mathrm{m}$ improvement ${ }^{24}$; and a 'within patient' achievement of the MCID for the SGRQ ( $>4$ point reduction) following PR. ${ }^{17}$ We corroborated these results by using receiver operator characteristic (ROC) curves to identify the change in 5STS with highest $\mathrm{C}$-statistic to discriminate between patients according to anchor cut-offs for the Likert scale, ISW and SGRQ.

\section{Statistical analysis}

Statistical analyses and graphical representations were made using GraphPad Prism 5.0 and SPSS V.21. Single measure intraclass correlation coefficients (ICCs) of consistency and random effect were calculated with $95 \%$ CIs to examine test-retest and interobserver reliability. A paired t test was performed to assess any learning effect between test and retest observations. BlandAltman plots were plotted to demonstrate the 95\% limits of agreement. For convergent validity, Spearman rank correlation was used to quantify the association between 5STS and other outcome measures. For discriminative validity (or validity by extreme groups), unpaired $t$ tests or non-parametric equivalent were used to compare between groups able and unable to perform the 5STS test. Pre- and post-PR measurements were compared by paired $\mathrm{t}$ tests or non-parametric equivalent. Substantially skewed data were log transformed before analysis to calculate effect sizes. Cohen's d was calculated to estimate effect size using the mean difference and SD before and after 
PR; this allowed an assessment of the magnitude of change with PR. A p value $<0.05$ was considered significant.

\section{RESULTS}

Reliability

The 5STS showed excellent test-retest and interobserver reliability with an ICC of 0.97 (95\% CI 0.95 to 0.99 ) and 0.99 (95\% CI 0.99 to 1.00 ), respectively. There was no significant difference in 5STS time recorded between test and retest observations with a mean difference of 0.04 (95\% CI -0.21 to 0.29$) \mathrm{s}$; $\mathrm{p}=0.78$. Bland-Altman plots are shown in figure 1 , demonstrating a bias of -0.00 and $-0.04 \mathrm{~s}$ for interobserver and test-retest measurement error, respectively.

\section{Convergent validity}

Baseline characteristics of the cross-sectional cohort are shown in table 1. Aside from age, there was no relationship between 5STS and anthropometric measures. The strongest relationship observed was a significant negative correlation between 5STS time and exercise capacity (as measured by ISW) (table 1 and figure 2). Inspection of figure 2 shows a curvilinear relationship (second-order polynomial) between 5STS and ISW (goodness of fit: $R^{2}=0.32$ ) with the curve flatter at low ISW values. Although, as expected, there was a negative relationship between 5STS time and lower limb muscle strength (as measured by QMVC and QMVC percentage predicted; table 1 and figure 2), this was not as strong as the relationship between 5STS and ISW.

There was also a positive association between slower 5STS time and worse health-related quality of life (higher SGRQ and CAT) and MRC dyspnoea score. Furthermore, increased 5STS time correlated significantly with worsening prognosis indices

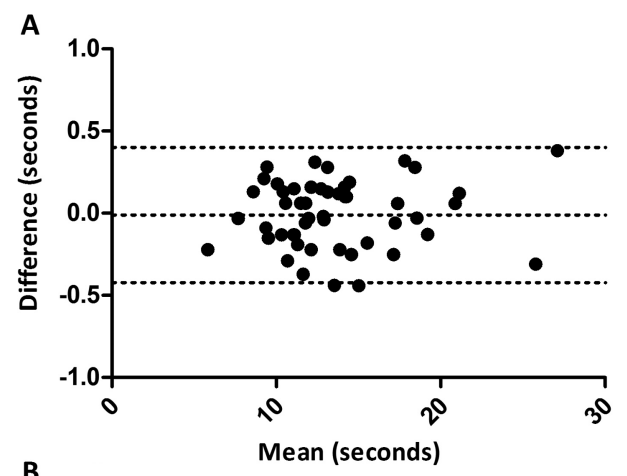

Upper Limit 0.40

Mean -0.00

Lower Limit $\mathbf{- 0 . 4 1}$

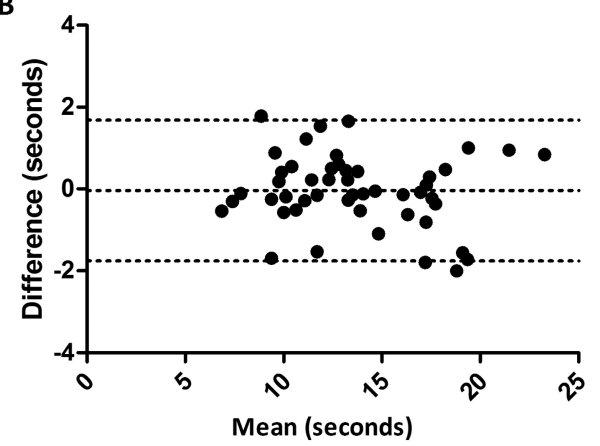

Upper Limit 1.69

Mean -0.04

Lower Limit -1.76

Figure 1 Bland-Altman plots for (A) interobserver and (B) test-retest reliability, with difference between measurements (y axis) plotted against mean of the measurements ( $x$ axis). The upper and lower dotted lines represent the $95 \%$ limits of agreement for the comparison while the middle dotted line represents the mean bias. The bias was -0.00 for interobserver measurement error and $-0.04 \mathrm{~s}$ for test-retest.
Table 1 Baseline characteristics expressed as mean (SD) and Spearman rank correlation with five-repetition sit-to-stand (5STS) manoeuvres

\begin{tabular}{lccr}
\hline Characteristic & Mean (SD) & Spearman rank & p Value \\
\hline Age & $69(10)$ & 0.15 & $<0.01$ \\
Sex (M/F) & $262 / 213$ & -0.06 & 0.24 \\
Height (m) & $1.66(0.10)$ & -0.08 & 0.09 \\
Weight (kg) & $77.0(21.7)$ & 0.00 & 0.95 \\
BMI (kg/m $\left.{ }^{2}\right)$ & $27.8(6.8)$ & 0.04 & 0.37 \\
FEV $\%$ predicted & $47.6(20.6)$ & -0.10 & 0.05 \\
ISW (m) & $203(145)$ & -0.59 & $<0.01$ \\
ISW (\% predicted) & $34(23)$ & -0.51 & $<0.01$ \\
QMVC (kg) & $24.6(9.5)$ & -0.33 & $<0.01$ \\
QMVC (\% predicted) & $57.8(16.9)$ & -0.38 & $<0.01$ \\
MRC & $3.5(1.1)$ & 0.43 & $<0.01$ \\
SGRQ & $53.6(16.6)$ & 0.35 & $<0.01$ \\
CAT & $22.2(7.9)$ & 0.31 & $<0.01$ \\
Charlson & $4.2(1.6)$ & 0.14 & $<0.01$ \\
ADO & $5.1(1.7)$ & 0.42 & $<0.01$ \\
iBODE & $4.6(2.6)$ & 0.46 & $<0.01$ \\
5STS (s) & $15.4(6.5)$ & 1.00 & $<0.01$ \\
\hline ADO Age & &
\end{tabular}

ADO, Age Dyspnoea Obstruction index; BMI, body mass index; CAT, COPD Assessment Test; Charlson, age-adjusted Charlson Index; FEV ${ }_{1}$, forced expiratory volume in $1 \mathrm{~s}$; iBODE, BODE index with incremental shuttle walk as measure of exercise capacity; ISW, incremental shuttle walk; MRC, Medical Research Council dyspnoea score; QMVC, quadriceps maximum voluntary contraction; SGRQ, St George's Respiratory Questionnaire.
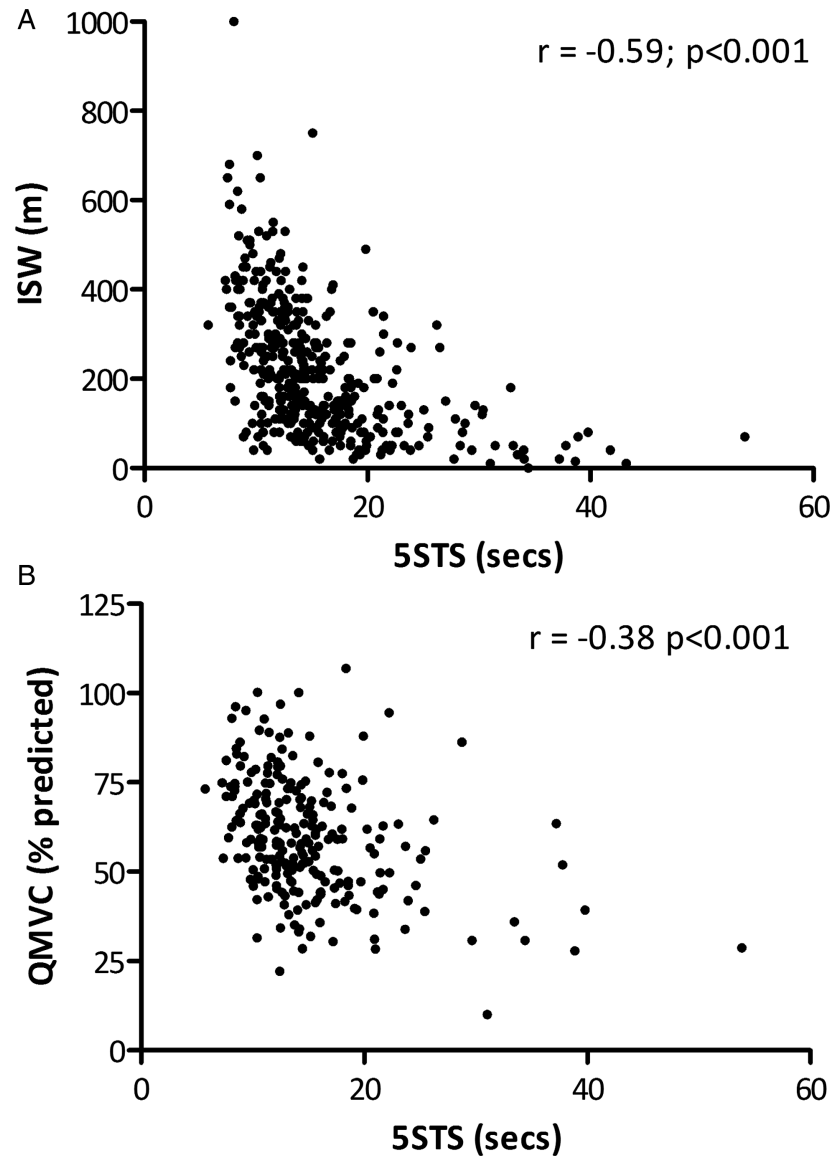

Figure 2 Relationship between the five-repetition sit-to-stand test (5STS) and (A) the incremental shuttle walk (ISW) test and (B) quadriceps maximum voluntary contraction (QMVC). 
score (higher ADO and iBODE). These relationships were moderate and in line with the strength of association between 5STS and lower limb muscle strength. No significant association was seen between forced expiratory volume in $1 \mathrm{~s}\left(\mathrm{FEV}_{1}\right.$ percentage predicted) and 5STS.

\section{Discriminative validity}

Seventy patients (15\% of total cohort) failed to complete the 5STS. The clinical characteristics of those who completed and those who failed to complete the 5STS are shown in table 2. Those who failed to complete the 5STS had very significantly reduced exercise capacity and quadriceps strength compared with those able to complete the test.

\section{Responsiveness to PR}

Of the 305 patients referred for PR, 18 failed to start, 40 failed to attend at least $50 \%$ of the supervised sessions and 8 completed but failed to attend post-PR assessment. Data from the remaining 239 participants (136 men) were analysed. Baseline characteristics, expressed as mean (SD), were age 70 (9) years, body mass index $27.5(6.1) \mathrm{kg} / \mathrm{m}^{2}, \mathrm{FEV}_{1}$ percentage predicted 48.1 (19.8) and MRC dyspnoea 3.3 (1.1).

Table 3 shows the changes and Cohen's d effect sizes in 5STS, ISW and SGRQ following PR. There was a significant reduction in median 5STS with PR $(-1.4 \mathrm{~s})$, which correlated significantly with change in ISW $(r=-0.13 ; \mathrm{p}<0.05)$. For those 'feeling much better' or 'better' after PR, the median (25th, 75th centiles) change in 5STS was $-1.5(-3.9,-0.2) \mathrm{s}$. The median (25th, 75th centiles) change in 5STS in those achieving the MCID in the ISW and SGRQ following PR was -1.7 (-4.4, $-0.3)$ and $-1.7(-3.7,-0.1) \mathrm{s}$, respectively. Using ROC plots, the change in 5STS that best discriminated patients 'feeling much better' or 'better' was $-1.3 \mathrm{~s}$, achieving the MCID in the ISW was $-1.4 \mathrm{~s}$ and achieving the MCID in the SGRQ was $-1.4 \mathrm{~s}$. Using the most conservative estimate for the MCID, the

Table 2 Comparison between patients with chronic obstructive pulmonary disease who completed or failed to complete the five-repetition sit-to-stand (5STS) manoeuvre

\begin{tabular}{lccr}
\hline Characteristic & \multicolumn{2}{l}{$\mathbf{5 S T S}$} & p Value \\
\cline { 2 - 3 } & $\begin{array}{l}\text { Completed } \\
\mathrm{n}=\mathbf{4 0 5}\end{array}$ & $\begin{array}{l}\text { Failed } \\
\mathbf{n}=\mathbf{7 0}\end{array}$ & \\
\hline Age (years) & $68(10)$ & $73(10)$ & $<0.01$ \\
Sex (M/F) & $222 / 183$ & $40 / 30$ & 0.80 \\
BMI (kg/m²) & $27.5(6.4)$ & $29.2(8.4)$ & 0.05 \\
FEV $(\%$ predicted) & $47.7(20.4)$ & $46.9(21.7)$ & 0.76 \\
MRC & $3.3(1.1)$ & $4.1(1.0)$ & $<0.01$ \\
ISW (m) & $224(146)$ & $84(66)$ & $<0.01$ \\
ISW (\% predicted) & $37(23)$ & $16(12)$ & $<0.01$ \\
QMVC (kg) & $25.6(9.4)$ & $17.6(6.7)$ & $<0.01$ \\
QMVC (\% predicted) & $59.6(16.5)$ & $44.3(13.5)$ & $<0.01$ \\
SGRQ & $53.0(16.4)$ & $57.5(17.0)$ & 0.05 \\
Charlson Index & $4.1(1.6)$ & $4.9(1.7)$ & $<0.01$ \\
ADO & $4.9(1.7)$ & $6.0(1.5)$ & $<0.01$ \\
iBODE & $4.3(2.6)$ & $6.2(2.2)$ & $<0.01$
\end{tabular}

Data expressed as mean (SD). Groups compared using unpaired $t$ test. ADO, Age Dyspnoea Obstruction index; BMI, body mass index; Charlson, age-adjusted Charlson Index; FEV 1 , forced expiratory volume in $1 \mathrm{~s}$; iBODE, BODE index with incremental shuttle walk as measure of exercise capacity; ISW, incremental shuttle walk; MRC, Medical Research Council dyspnoea score; QMVC, quadriceps maximum voluntary contraction; SGRQ, St George's Respiratory Questionnaire.
Table 3 Response to pulmonary rehabilitation (PR)

\begin{tabular}{llcll}
\hline Outcome & Pre-PR & Change with PR & Effect size & p Value \\
\hline 5STS (s) & $14.1(11.5,21.3)$ & $-1.4(-3.9,0.0)$ & 0.32 & $<0.01$ \\
ISW (m) & $200(80,340)$ & $50(10,100)$ & 0.46 & $<0.01$ \\
SGRQ Total & $52.2(16.4)$ & $-4.7(12.0)$ & 0.22 & $<0.01$
\end{tabular}

Data expressed as median (25th, 75th centiles) or mean (SD).

ISW, incremental shuttle walk; SGRQ, St George's Respiratory Questionnaire; 5STS, five-repetition sit-to-stand test.

proportion of patients in whom the 5STS was reduced by $\geq 1.7$ s with $\mathrm{PR}$ was $44 \%$, similar to the proportion achieving an improvement in MCID in the ISW $(50 \% ; p=0.20$, Fisher exact test).

\section{DISCUSSION}

We have demonstrated that the 5STS shows good test-retest and interobserver reliability in COPD. In a cohort of patients with stable COPD, 5STS was significantly correlated with measures of exercise capacity, lower limb strength, health-related quality of life and dyspnoea, supporting convergent validity for the measure. Stratification according to failure to complete the 5STS identified significant impairment in exercise capacity and profound quadriceps muscle weakness, supporting discriminative validity. Furthermore, we were able to demonstrate that the 5STS is responsive to PR in COPD and have proposed a MCID, suggesting potential utility as an outcome measure.

\section{Reliability and validity of 5STS in COPD}

Previous data on the STS manoeuvre in patients with COPD are limited to a few studies. Roig et $a l^{25}$ measured the 5STS in 21 patients with stable COPD and 21 healthy age-matched controls and showed that patients with COPD needed 21\% more time to complete the 5STS than controls. However, they were not able to demonstrate any significant relationship between 5STS and knee extensor muscle strength or muscle cross-sectional area as measured by CT, perhaps due to the small sample size. In a convenience sample of 53 patients with COPD, Ozalevli et $a l^{9}$ demonstrated a significant relationship between the maximum number of STS manoeuvres performed in 1 min and 6 min walk test and quadriceps muscle strength. The 5STS is also noted to be a single component of the short physical performance battery (SPPB), a standardised objective tool which consistently identifies poor prognosis in community-dwelling older adults. ${ }^{26}$ The SPPB has been studied in a single cohort of relatively young COPD patients with milder disease, demonstrating lower scores compared with age-matched healthy controls and associations with lung function, body composition and increased risk of disability. ${ }^{27-29}$

Apart from the considerably larger sample size, our data adds to the current literature by demonstrating the test-retest and interobserver reliability of the 5STS in patients with COPD. The ICCs were high in our study and in line with the results from a systematic review by Bohannon ${ }^{11}$ of 10 studies performed largely in community-dwelling older adults who reported test-retest ICC ranging from 0.64 to 0.96 . We also demonstrated a strong correlation between 5STS and maximum exercise performance as measured by ISW, supporting the potential use of the 5STS as a simple functional outcome measure. Although the 5STS is purported to be a measure of lower limb strength in older adults, we found only a modest (but significant) relationship with quadriceps strength. A possible 
explanation is that, in patients with COPD, factors other than lower limb muscle strength, such as impaired lung function or balance, have a more significant influence on 5STS performance than in health community-dwelling older adults.

Interestingly, we were also able to show significant correlations between 5STS and validated prognostic indices (iBODE and $\mathrm{ADO}$ ), established health-related quality of life measures and dyspnoea, further supporting its potential value as a global marker of COPD disease severity. This significant association with established indices of prognosis in COPD—such as ISW, $\mathrm{QMVC}^{31} \mathrm{iBODE}^{20}$ and $\mathrm{ADO}^{21}$ — suggests that the 5STS may also have value as a prognostic marker, and preliminary longitudinal studies appear to support this hypothesis. ${ }^{32}$

\section{Responsiveness of 5STS in COPD}

As the STS manoeuvre is related to lower limb muscle function and reflects an important activity of daily living, previous training studies in patients with COPD have used this as an outcome measure. However, due to the heterogeneity of the population and interventions and the small sample sizes, data regarding the responsiveness of the STS in COPD have been inconsistent. Kongsgaard et $a l^{33}$ randomised elderly men with COPD to 12 weeks of resistance training or control. There was no significant improvement in the number of STS manoeuvres completed in $30 \mathrm{~s}$ in either group, although only six patients completed resistance training. Two studies have used STS to evaluate the effect of additional resistance training in patients with COPD undergoing traditional aerobic-based PR. Panton et $a l^{34}$ demonstrated a significant between-group improvement in the number of increased STS manoeuvres in $1 \mathrm{~min}$ in the additional resistance group, while Phillips $e t a l^{35}$ showed no between-group differences in the number of STS repetitions. More recently, Gloeckl et $a l^{36}$ randomised 72 patients to a supervised squat exercise programme with or without additional whole body vibration, and showed significant improvements in the 5STS within both groups but no between-group differences.

In the current study we were able to demonstrate a significant improvement in 5STS time in a large COPD cohort undergoing outpatient PR, which correlated with change in ISW. The effect size was modest $(\mathrm{d}=0.32)$ and somewhere between the SGRQ and ISW, measures which are routinely used in PR. Interestingly, despite using different clinical anchors (patient self-report, exercise capacity, health-related quality of life) and different anchorbased approaches, we obtained a consistent estimate of between $1.3 \mathrm{~s}$ and $1.7 \mathrm{~s}$ for the MCID of the 5STS.

\section{Advantages and limitations of the 5STS in COPD}

There are obvious advantages to the 5STS as an assessment tool in COPD. First, it is quick to perform (all patients completed the test within $2 \mathrm{~min}$ ) and our data suggest there is no learning effect. This contrasts with the 6MWT and the ISW, both of which require repeat walks with adequate rest between tests (usually $30 \mathrm{~min}$ ). ${ }^{2}{ }^{3}$ Second, the 5 STS is cheap to perform with easily available equipment (chair and stopwatch). Third, the test requires only limited space, which makes it feasible in most healthcare settings including the home setting.

The major limitation to the test is the presence of a 'floor' effect, with up to $15 \%$ of our cohort unable to attempt or complete the test. Hence, the 5STS may have increased value as a functional outcome measure in better functioning patients. Other simple functional outcome tests, such as the habitual gait speed over $4 \mathrm{~m},{ }^{37}$ or a battery of physical performance measures, such as the SPPB, ${ }^{38}$ may be more appropriate for more poorly functioning individuals. We propose that the 5STS may be particularly useful as a functional outcome tool in certain healthcare settings, such as the outpatient clinic or home setting, where space and equipment may be at a premium.

Another potential use for the 5STS is as a stratification tool. Our data showed that patients unable to complete the 5STS had grossly reduced exercise capacity and considerable quadriceps weakness (table 2, figure 2), and we propose that the 5STS could be used as a simple bedside or clinic assessment tool to identify significantly impaired walking capacity or lower limb weakness. For example, the risk of mortality increases markedly when ISW falls below $170 \mathrm{~m}^{30}$; using receiver-operator characteristic curves plotted for our cohort, the 5STS had a C-statistic (area under the curve) of 0.82 to identify an ISW below $170 \mathrm{~m}$.

In summary, the 5STS is reliable, correlates with exercise capacity and quadriceps strength and is responsive to PR in COPD. It is a practical functional outcome measure suitable for use in most healthcare settings.

Acknowledgements The authors would like to acknowledge the Harefield Pulmonary Rehabilitation Unit at Harefield Hospital for their assistance in collecting the data.

Contributors SEJ, SSCK, JLC, MSP and CMN recruited patients and collected the data. SEJ and SSCK performed the analysis of data and preparation of the first draft of the manuscript. All authors contributed to the design of the study. WD-CM conceived the idea and is the guarantor of the paper, taking responsibility for the integrity of the work as a whole, from inception to published article.

Funding SEJ is supported by the Collaboration for Leadership in Applied Health Research and Care (CLAHRC) for northwest London. SSCK is supported by the Medical Research Council (MRC). JLC is supported by the NIHR Respiratory Biomedical Research Unit at the Royal Brompton and Harefield NHS Foundation Trust and Imperial College London. WD-CM is supported by a National Institute for Health Research Clinician Scientist Award (CS/7/007), a Medical Research Council (UK) New Investigator Research Grant (G1002113) and a National Institute for Health Research Clinical Trials Fellowship (NIHR-CTF-01-12-04). This project was undertaken at the NIHR Respiratory Biomedical Research Unit at the Royal Brompton and Harefield NHS Foundation Trust and Imperial College London; MIP's salary is part funded by the Biomedical Research Unit. The views expressed in this publication are those of the authors and not necessarily those of the NHS, The National Institute for Health Research nor the Department of Health.

Competing interests None.

Ethics approval All participants gave informed consent and the study was approved by the West London and the London-Camberwell St Giles research ethics committees (11/H0707/2 and 11/LO/1780).

Provenance and peer review Not commissioned; externally peer reviewed.

\section{REFERENCES}

1 Spruit MA, Polkey MI, Celli B, et al. Predicting outcomes from 6-minute walk distance in chronic obstructive pulmonary disease. J Am Med Dir Assoc 2012;13:291-7.

2 American Thoracic Society. ATS statement: guidelines for the six-minute walk test. Am J Respir Crit Care Med 2002;166:111-17.

3 Singh SJ, Morgan MD, Scott $S$, et al. Development of a shuttle walking test of disability in patients with chronic airways obstruction. Thorax 1992;47:1019-24.

4 Dall PM, Kerr A. Frequency of the sit to stand task: an observational study of free-living adults. App/ Ergon 2010;41:58-61.

5 Bohannon RW. Body weight-normalized knee extension strength explains sit-to-stand independence: a validation study. J Strength Cond Res 2009;23:309-11.

6 Lord SR, Murray SM, Chapman K, et al. Sit-to-stand performance depends on sensation, speed, balance, and psychological status in addition to strength in older people. J Gerontol A Biol Sci Med Sci 2002;57:M539-43.

7 Bohannon RW. Reference values for the five-repetition sit-to-stand test: a descriptive meta-analysis of data from elders. Percept Mot Skills 2006;103:215-22.

8 Jones CJ, Rikli RE, Beam WC. A 30-s chair-stand test as a measure of lower body strength in community-residing older adults. Res Q Exerc Sport 1999;70:113-19.

9 Ozalevli S, Ozden A, Itil 0, et al. Comparison of the sit-to-stand test with 6 min walk test in patients with chronic obstructive pulmonary disease. Respir Med 2007;101:286-93.

10 Schaubert KL, Bohannon RW. Reliability and validity of three strength measures obtained from community-dwelling elderly persons. J Strength Cond Res 2005; 19:717-20

11 Bohannon RW. Test-retest reliability of the five-repetition sit-to-stand test: a systematic review of the literature involving adults. J Strength Cond Res 2011;25:3205-7. 
12 McCarthy EK, Horvat MA, Holtsberg PA, et al. Repeated chair stands as a measure of lower limb strength in sexagenarian women. J Gerontol A Biol Sci Med Sci 2004:59:1207-12.

13 Rabe KF, Hurd S, Anzueto A, et al. Global strategy for the diagnosis, management, and prevention of chronic obstructive pulmonary disease: GOLD executive summary. Am J Respir Crit Care Med 2007;176:532-55.

14 Jones SE, Kon SS, Canavan JL, et al. Five-repetition sit-to-stand test: reliability, validity and response to pulmonary rehabilitation in COPD. Thorax 2012;67(Suppl 2):A53.

15 Man WD, Hopkinson NS, Harraf F, et al. Abdominal muscle and quadriceps strength in chronic obstructive pulmonary disease. Thorax 2005;60:718-22.

16 Seymour JM, Spruit MA, Hopkinson NS, et al. The prevalence of quadriceps weakness in COPD and the relationship with disease severity. Eur Respir J 2010;36:81-8

17 Jones PW, Quirk FH, Baveystock CM. The St George's respiratory questionnaire. Respir Med 1991;85(Suppl B):25-31.

18 Dodd JW, Hogg L, Nolan J, et al. The COPD assessment test (CAT): response to pulmonary rehabilitation. A multicentre, prospective study. Thorax 2011;66:425-9.

19 Fletcher CM, Elmes PC, Fairbairn AS, et al. The significance of respiratory symptoms and the diagnosis of chronic bronchitis in a working population. Br Med J $1959 ; 2: 257-66$.

20 Williams JE, Green RH, Warrington V, et al. Development of the i-BODE: validation of the incremental shuttle walking test within the BODE index. Respir Med 2012:106:390-6.

21 Puhan MA, Garcia-Aymerich J, Frey M, et al. Expansion of the prognostic assessment of patients with chronic obstructive pulmonary disease: the updated BODE index and the ADO index. Lancet 2009;374:704-11.

22 Charlson ME, Pompei $\mathrm{P}$, Ales $\mathrm{KL}$, et al. A new method of classifying prognostic comorbidity in longitudinal studies: development and validation. J Chronic Dis 1987:40:373-83.

23 Revicki D, Hays RD, Cella D, et al. Recommended methods for determining responsiveness and minimally important differences for patient-reported outcomes. J Clin Epidemiol 2008;61:102-9.

24 Singh SJ, Jones PW, Evans R, et al. Minimum clinically important improvement for the incremental shuttle walking test. Thorax 2008;63:775-7.

25 Roig M, Eng JJ, MacIntyre DL, et al. Deficits in muscle strength, mass, quality, and mobility in people with chronic obstructive pulmonary disease. J Cardiopulm Rehabil Prev 2011;31:120-4
26 Guralnik JM, Simonsick EM, Ferrucci L, et al. A short physical performance battery assessing lower extremity function: association with self-reported disability and prediction of mortality and nursing home admission. J Gerontol 1994;49:M85-94.

27 Eisner MD, Blanc PD, Sidney $S$, et al. Body composition and functional limitation in COPD. Respir Res 2007:8:7.

28 Eisner MD, Iribarren C, Blanc PD, et al. Development of disability in chronic obstructive pulmonary disease: beyond lung function. Thorax 2011;66:108-14

29 Eisner MD, Iribarren C, Yelin EH, et al. Pulmonary function and the risk of functional limitation in chronic obstructive pulmonary disease. Am J Epidemiol 2008;167:1090-101.

30 Ringbaek T, Martinez G, Brondum E, et al. Shuttle walking test as predictor of survival in chronic obstructive pulmonary disease patients enrolled in a rehabilitation program. J Cardiopulm Rehabil Prev 2010;30:409-14.

31 Swallow EB, Reyes D, Hopkinson NS, et al. Quadriceps strength predicts mortality in patients with moderate to severe chronic obstructive pulmonary disease. Thorax 2007:62:115-20

32 Puhan MA, Siebeling L, Zoller M, et al. Simple functional performance tests and mortality in COPD. Eur Respir J. Published Online First: 21 Mar 2013. doi:10.1183/ 09031936.00131612

33 Kongsgaard $M$, Backer $\mathrm{V}$, Jorgensen $\mathrm{K}$, et al. Heavy resistance training increases muscle size, strength and physical function in elderly male COPD patients - a pilot study. Respir Med 2004;98:1000-7.

34 Panton LB, Golden J, Broeder CE, et al. The effects of resistance training on functional outcomes in patients with chronic obstructive pulmonary disease. Eur J Appl Physiol 2004;91:443-9.

35 Phillips WT, Benton MJ, Wagner $\mathrm{CL}$, et al. The effect of single set resistance training on strength and functional fitness in pulmonary rehabilitation patients. J Cardiopulm Rehabil 2006;26:330-7.

36 Gloeckl R, Heinzelmann I, Baeuerle $S$, et al. Effects of whole body vibration in patients with chronic obstructive pulmonary disease - a randomized controlled trial. Respir Med 2012;106:75-83.

37 Kon SS, Patel MS, Canavan JL, et al. Reliability and validity of the four metre gait speed in COPD. Eur Respir J. Published Online First: 6 Dec 2012. doi:10.1183/ 09031936.00162712

38 Latham NK, Mehta V, Nguyen AM, et al. Performance-based or self-report measures of physical function: which should be used in clinical trials of hip fracture patients? Arch Phys Med Rehabil 2008;89:2146-55. 